\title{
An analysis of deep vein thrombosis in 1277 consecutive neurosurgical patients undergoing routine weekly ultrasonography
}

\author{
Clinical article
}

\author{
Akil P. Patel, M.D., ${ }^{1}$ Michael T. Koltz, M.D., ${ }^{1}$ Charles A. Sansur, M.D., M.H.Sc., 1 \\ Mangla Gulati, M.D., ${ }^{2}$ and D. Kojo Hamilton, M.D. ${ }^{3}$ \\ Departments of ${ }^{1}$ Neurological Surgery and ${ }^{2}$ Medicine, University of Maryland School of Medicine, \\ Baltimore, Maryland; and ${ }^{3}$ Department of Neurological Surgery, Oregon Health \& Science \\ University, Portland, Oregon
}

Object. Patients requiring neurosurgical intervention are known to be at increased risk for deep vein thrombosis (DVT) and attendant morbidity and mortality. Pulmonary embolism (PE) is the most catastrophic sequela of DVT and is the direct cause of death in $16 \%$ of all in-hospital mortalities. Protocols for DVT screening and early detection, as well as treatment paradigms to prevent PE in the acute postoperative period, are needed in neurosurgery. The authors analyzed the effectiveness of weekly lower-extremity venous duplex ultrasonography (LEVDU) in patients requiring surgical intervention for cranial or spinal pathology for detection of DVT and prevention of PE.

Methods. Data obtained in 1277 consecutive patients admitted to a major tertiary care center requiring neurosurgical intervention were retrospectively reviewed. All patients underwent admission (within 1 week of neurosurgical intervention) LEVDU as well as weekly LEVDU surveillance if the initial study was normal. Additional LEVDU was ordered in any patient in whom DVT was suspected on daily clinical physical examination or in patients in whom chest CT angiography confirmed a pulmonary embolus. An electronic database was created and statistical analyses performed.

Results. The overall incidence of acute DVT was 2.8\% (36 patients). Of these cases of DVT, a statistically significant greater number $(86 \%)$ were discovered on admission (within 1-7 days after admission) screening LEVDU (p $<0.05)$, whereas fewer were documented 8-14 days after admission (2.8\%) or after 14 days (11.2\%) postadmission. Additionally, for acute DVT detection in the present population, there were no underlying statistically significant risk factors regarding baseline physical examination, age, ambulatory status, or type of surgery.

The overall incidence of acute symptomatic PE was $0.3 \%$ and the mortality rate was $0 \%$.

Conclusions. Performed within 1 week of admission in patients who will undergo neurosurgical intervention, LEVDU is effective in screening for acute DVT and initiating treatment to prevent PE, thereby decreasing the overall mortality rate. Routine LEVDU beyond this time point may not be needed to detect DVT and prevent PE unless a change in the patient's physical examination status is detected.

(http://thejns.org/doi/abs/10.3171/2012.11.JNS121243)

\section{KEY WORDS - deep vein thrombosis}

\section{- ultrasonography}

\section{pulmonary embolism}

$\mathrm{D}$ EEP vein thrombosis, a subset disease of venous thromboembolism, is a major cause of morbidity and mortality in all patients. Lower-extremity DVTs are believed to be the source of as many as $90 \%$ of pulmonary emboli, many with fatal outcomes. ${ }^{7}$ Pulmonary embolism is the leading cause of preventable deaths in hospitalized patients, causing, if untreated, death in as many as $16 \%$ of hospitalized patients. ${ }^{13}$ In regard to venous thromboembolism, patients undergoing neurological surgery are considered to be at moderate risk..$^{13}$ The rate of DVT formation associated with cranial and neurosurgical spinal procedures performed without prophylactic measures varies from $29 \%$ to $43 \%$., 3,16 The reported incidence of DVT in untreated neurosurgical patients varies

Abbreviations used in this paper: DVT = deep vein thrombosis; $\mathrm{IVC}=$ inferior vena cava; $\mathrm{PE}=$ pulmonary embolism. between $18 \%$ and $50 \% .^{1,12,17}$ While mechanical devices for DVT prophylaxis are considered to be a standard of care,, 14 chemical prophylaxis has had the greatest impact (45\% relative risk reduction in venous thromboembolism based on one meta-analysis). ${ }^{20,29,31}$ Nonetheless, incidence rates reflecting poor outcomes continue to stay fairly high. ${ }^{17,24}$ Various guidelines have been published to better screen and treat DVTs promptly and efficiently. However, there are no set universal protocols that have showed true significant success. ${ }^{4,5}$ This is especially true for practices involving routine screening ultrasonography. ${ }^{2,15,23}$ While this modality is not as sensitive for diagnosing DVT as the gold standard of lower-extremity venography, ${ }^{14}$ it has certainly helped in earlier and increased detection of DVTs. ${ }^{8,11,19}$ What remains unclear is when and how often these ultrasound studies should be performed to maximize DVT screening and detection, as well as possibly improve 
PE outcomes. Is there validity to performing routine ultrasonography in all patients, regardless of functional status, compared with those who have neurological deficits leading to severe paresis/plegia, restricting mobility?

In the present study, over a period of 1 year, we investigated, in an acute inpatient setting, the usefulness of routine screening ultrasonography in all neurosurgical patients and determined whether there are any differences between the various subsets of the population. To ascertain if there is an optimal time window for DVT screening, we also analyzed the frequency and duration of ultrasound surveillance.

\section{Methods}

After receiving approval for the study, including patient consent status, from our institutional research review board, we conducted an extensive literature search to determine the current guidelines for DVT screening and ultrasound findings. This was followed by a retrospective review of prospectively gathered data of all adult patients who were admitted to the acute inpatient center at the University of Maryland Medical Center for any neurosurgical procedure during a 1-year period (2010-2011). Patient demographic information, admission/discharge diagnosis, type of surgery, and duration of stay were recorded. Inclusion criteria included a screening ultrasound study on admission and weekly surveillance ultrasonography thereafter of the lower extremities for the duration of the patient's hospital stay, regardless of the patient's overall risk assessment for likelihood of developing DVT. Any patient in whom a negative initial ultrasound result was obtained and who then developed clinical symptoms or signs of DVT, such as calf swelling/tenderness, redness, warmth, acute edema, or weakness, underwent an immediate diagnostic study for possible DVT. These patients were not excluded from the study. The total number of ultrasound studies and the number of positive lowerextremity DVT studies were recorded and analyzed. Patients with preexisting acute DVT were excluded from the screening ultrasound, and also those with solely chronic DVT were excluded from the overall study analysis.

With regard to perioperative prophylaxis, all patients had pneumatic compression devices placed before, during, and after surgery. Patients who were in-house prior to surgery additionally received subcutaneous heparin; however, the midnight dose prior to the day of surgery was withheld. In all patients, weight-based subcutaneous heparin was initiated 24 hours postoperatively, unless a strong contraindication, such as active bleeding or heparin-induced thrombocytopenia, existed. All spine patients also underwent early postoperative mobilization with the aid of daily physical/occupational therapy.

There was also a specific protocol put forth for DVTs found on duplex screening in contrast to symptomatic thrombophlebitis or PE. For the treatment of DVT found on routine duplex screening, the interventional radiology team was consulted for placement of a percutaneous IVC filter. This was almost always done on the same day of detection. In comparison, patients with a positive duplex study in addition to symptomatic thrombophlebitis or PE immediately underwent anticoagulation in the form of a heparin drip. The heparin drip was monitored via routine blood withdrawals to determine prothrombin time, with an overall goal of 60-80 seconds. After this, the interventional radiology team placed an IVC filter.

Each case was counted only once for DVT occurrence during the entire study period. Cases involving concurrent acute and chronic DVT were also counted once.

An electronic database generated 1277 neurosurgical patients who had undergone screening ultrasonography over the course of the year. The database was then set up to highlight those patients in whom DVT was diagnosed during their hospital stay and in whom it was confirmed with ultrasound screening.

Statistical analyses of these data were performed using SPSS for Windows v15.0 (SPSS Inc.). The chi-square or Fisher exact test was used for categorical data depending on the number of patients in each group. The twosample unpooled t-test with unequal variance was used for analysis of the two means. All tests were two tailed. Multivariate analysis was performed using binomial logistic regression analysis. Differences were considered statistically significant for $\mathrm{p}<0.05$.

\section{Results}

A total of 1277 patients underwent lower-extremity ultrasound screening at our center over the course of 1 year. Patient demographics, sex, and age were reviewed and recorded. Of these patients, 40 (3.1\%) were found to have proximal and/or distal DVT. The overall mean age in the $22(55 \%)$ male and 18 female $(45 \%)$ patients was 61.9 years (range $40-82$ years). Diagnoses included 32 patients with acute DVT, 4 with chronic DVT, and 4 with both acute and chronic DVT. Based on the literature, a 1-month cutoff was used to distinguish acute from chronic DVT. To determine the most effective timing for DVT screening, patients with only chronic DVTs were not included in the overall data analysis. Twenty-seven patients underwent cranial procedures and 13 underwent spinal procedures $(\mathrm{p}>0.05)$. Among the 686 cranial surgery patients, the incidence of DVT was $1.9 \%(\mathrm{p}<0.05)$. Among the 591 spine surgery patients, the incidence of DVT was 2.2\% (p <0.05). Based on multiple documented examinations, 15 of the 40 patients were densely paretic, 0 were plegic, and 25 had full-strength motor examination results $(\mathrm{p}>0.05$ for acute DVT vs $\mathrm{p}<0.05$ for all DVT diagnoses). We further evaluated the overall activity levels of these patients while at the hospital and found that 22 of the 40 patients had active orders for bed rest exceeding 48 hours related to their medical treatment, whereas 18 were cleared to be out-of-bed as tolerated ( $p$ $>0.05$ ). With regard to chemical prophylaxis, 34 patients were receiving subcutaneous heparin (5000 U thrice daily) or prophylactic treatment with Lovenox (Table 1). In all 40 patients, upon further review, the results of testing were negative for any blood clotting history or any hematological diagnosis that would predispose them to DVTs.

With regard to overall treatment and hospital course, 28 of the 40 patients received IVC filters after being di- 
Deep vein thrombosis in patients undergoing ultrasonography

TABLE 1: Summary of data obtained in 40 patients with DVT*

\begin{tabular}{lc}
\multicolumn{1}{c}{ Characteristic } & No. of Cases $(\%)$ \\
\hline sex & $18(45)$ \\
female & $22(55)$ \\
male & $61.9(40-82)$ \\
mean age in yrs (range) & \\
DVT at diagnosis & $32(80)$ \\
acute & $4(10)$ \\
chronic & $4(10)$ \\
acute \& chronic & \\
type of surgery & $27(67.5)$ \\
cranial & $13(32.5)$ \\
spinal & \\
motor exam status at diagnosis & $15(37.5)$ \\
paretic & $0(0)$ \\
plegic & $25(62.5)$ \\
normal & \\
activity status at time of diagnosis & $22(55)$ \\
bed rest ordered & $18(45)$ \\
out-of-bed ordered & \\
chemical prophylaxis begun at diagnosis & $34(85)$ \\
yes & $6(15)$ \\
no & $28(70)$ \\
\hline IVC filter postdiagnosis &
\end{tabular}

* All values reflect the number of cases (\%) based on a total of 40 , except for those of age, which represent years.

agnosed with acute or chronic DVT. While there were not any specific inclusion or exclusion criteria pertaining to patients who also concomitantly had a pulmonary embolus, 7 of the 40 patients were found to have both entities. Of these 7, 3 were diagnosed with chronic PE. There was a $0 \%$ mortality rate for these patients.

In analyzing the timing of DVT diagnosis in relation to hospital stay, of the 36 acute cases, 31 (86\%) were diagnosed with a DVT within the first 7 days of hospital stay, $1(2.8 \%)$ was diagnosed between 7 and 14 days of hospital stay, and $4(11.2 \%)$ were diagnosed after being in the hospital for more than 14 days $(\mathrm{p}<0.05)$. The average length of stay for the entire database was 7.2 days whereas that of the DVT group was 15.4 days $(\mathrm{p}<0.05)$.

Cases were also further analyzed with regard to the types of procedures they underwent for their specific diagnoses and physical statuses. There did not seem to be a strong, consistent correlation/pattern between one's physical activity status/capability and the likelihood of suffering a DVT immediately after neurosurgery. When comparing these results with timing of DVT detection in one's hospital stay, we did note that patients with dense paresis were likely to have symptomatic DVT findings.

\section{Discussion}

Neurosurgical patients have an increased risk of de- veloping DVT and PE due to limb paralysis, stroke, immobility, and hypercoagulable states often induced by neoplasms, trauma, and degenerative and iatrogenic causes. ${ }^{10,17,18,22,27}$

The reported incidence of DVT in the neurosurgical population varies drastically. A recent study, which followed a biweekly ultrasonography screening protocol, revealed an overall DVT incidence of $9.7 \%$, which was compiled from 237 cranial and spinal patients. ${ }^{19}$ This particular study's protocol was to administer chemical prophylaxis with unfractionated heparin $5000 \mathrm{U}$ twice daily and mechanical prophylaxis with pneumatic compression sleeves within 24 hours of admission.

Nurmohamed et al. ${ }^{26}$ also examined the incidence of DVT in neurosurgical patients and found a considerably higher incidence at $19 \%$. Their patients received lowmolecular-weight heparin and graduated compression stockings. Likewise, Agnelli et al. ${ }^{1}$ reported an overall DVT incidence of $17 \%$ in neurosurgical patients treated with both low-molecular-weight heparin and compression stockings who were tested on treatment Day 8, as determined by hospital protocol. In contrast, Misra et al. ${ }^{25}$ specifically focused on patients in the neurological ICU compared with other ICUs. While they did not have stringent inclusion criteria with regard to chemical and mechanical prophylaxis, they found an overall neurological ICU incidence of DVT of $2.55 \%$, significantly less than that in other DVT studies.

While our data do not relect a very high DVT rate, our findings were certainly statistically significant and similar to those of the studies above. However, while these studies focused on overall incidence, we wanted to further determine whether routine weekly screening with ultrasonography was truly justified in neurosurgical practice. We therefore made weekly ultrasound studies an absolute inclusion criterion for all 1277 consecutive patients we reviewed from our prospectively collected data.

Our study showed that a significant percentage (86\%) of patients with DVT were diagnosed within the 1st week of hospital stay. This finding is similar to that reported in a recent case review study in which the authors found that $84 \%$ of their patients with DVT were diagnosed with ultrasonography in the 1st week of hospital stay. ${ }^{21}$ The authors' subsequent biweekly surveillance studies had a drastic drop-off in terms of diagnosing DVTs at a later time during the hospital stay. Their study retrospectively examined the DVT data at their institution. In comparison, we analyzed prospectively gathered data over the course of 1 year to essentially verify the same findings. Our study also showed a drastic drop-off after the 1st week of surveillance and perhaps additional screening would benefit high-risk or symptomatic patients.

The significance of routine screening ultrasonography in neurosurgical patients has certainly been evaluated in previous studies and reports. Ray et al. ${ }^{27}$ focused on screening ultrasonography in patients with subarachnoid hemorrhage. Their study's overall DVT incidence for screened and unscreened patients was $18 \%$. This included all patients with subarachnoid hemorrhage who had undergone surgical or endovascular treatment and survived for more than 15 days. The investigators then focused only on asymptomatic patients who had undergone 
screening ultrasonography and determined an incidence of $24 \%$. Based on their analysis, ultrasound-screened patients were more likely to be diagnosed with DVT, but there was no statistical difference in the overall incidence of DVT or PE in screened and unscreened patients.

Another study that focused on routine ultrasonography as a diagnostic modality for DVT showed that $50 \%-$ $60 \%$ of high-risk patients with DVT may not have clinical manifestations such as a swollen, tender, warm calf, or a positive Homan sign. ${ }^{7}$ Therefore, screening ultrasonography would cover these patients, whereas in patients who had not undergone screening, such manifestations would have been missed.

Based on our findings, we recommend that continuing admission ultrasound studies be undertaken in all neurosurgical patients, as this truly provides a baseline study while preventing the possibility of overtreating patients with stable chronic DVT. A follow-up study within 1 week also appears to be very effective. Whether treatment is elective or emergent, cranial or spinal, and whether the patients are paretic or functionally intact, their recovery time still often plays a major role in overall ambulatory status within the 1st week of hospital stay, emphasizing the dire need to increase and encourage ambulation. In examining our overall lengths of stay, it is certain that the added morbidity of DVTs has played a role in the $100 \%$ increase in length of stay for patients with DVTs. After the initial week of stay, however, follow-up surveillance studies do not seem very helpful for all patients. Examining those patients who are symptomatic at this time, with an emphasis on the paretic/plegic population, would be the better option.

Our findings also further elaborate on overall PE outcomes. Pulmonary embolism is the one complication that all institutions attempt to avoid because of the high associated morbidity and mortality rates. Detecting and/ or preventing DVTs, which usually have straightforward treatments, is a means of avoiding the more serious complications of PE. We observed a $0.3 \%$ incidence of acute, symptomatic PE and a $0 \%$ mortality rate. Our incidence rate is similar to the $0.4 \%-2.0 \%$ rate reported in the neurosurgery literature..$^{16,20,30}$ In regard to mortality rates, while there are no major meta-analyses of PE-associated death in the cranial population, a recent meta-analysis of a spine population demonstrated a rate of $0.01 \% .{ }^{28} \mathrm{Of}$ 107,996 patients who underwent some form of spine surgery, 11 deaths due to PE were reported. Including the presumed PE cases, the rate rose to $0.02 \%$. We therefore believe that our $0 \%$ mortality rate validates the point that if DVT screening is performed during the 1st week of hospital stay, which was found to be statistically significant, then not only do we note a higher detection of DVTs earlier, but we also ultimately see lower overall PE and mortality rates. This is definitely a positive and successful quality measure for a major medical center.

We do recognize that while our results imply changing important aspects of core medical measures, there are obvious weaknesses to the study. The sample size, singleinstitution patient population, and the 1-year timeline to collect the data are certainly partial pitfalls. Because of the routine nature of the screening test and because our mixed-acuity inpatient census allowed for more urgent inpatient needs, our protocol allowed for inclusion of patients who underwent their scans after 48 hours postadmission but before 7 days postadmission. Less than $8 \%$ of the total population fell into this category, and none were among the patients who were positive for DVTs. Additionally, we do not have a true comparison arm with which to evaluate the effectiveness of routine, weekly DVT screening on a long-term basis against screening during a very specific period of time. More particularly, we are not able to compare the overall PE outcomes for these two specific groups. This also holds true when analyzing the current literature, which does not have any major retrospective or prospective comparison studies.

\section{Conclusions}

This is the first single-center study showing prospective DVT data that were retrospectively analyzed for routine ultrasound screening of all neurosurgical patients over a given period. We found that a significant proportion of DVTs were identified and treated within the 1st week after admission. Beyond the 1st week, lowerextremity venous duplex ultrasonography aids mostly in confirming the clinical diagnosis of symptomatic highrisk patients. Screening during this specific window not only effectively detects DVTs but, more significantly, allows us to detect and treat possible pulmonary emboli at an earlier time. Follow-up long-term ultrasound studies should only be considered for symptomatic patients, with a lower threshold of suspicion for paretic and/or plegic patients. The overall physical status, age, or activity level of the patient does not seem to have an influence on the acute setting and therefore should not play a role in initial screening.

\section{Disclosure}

The authors report no conflict of interest concerning the materials or methods used in this study or the findings specified in this paper.

Author contributions to the study and manuscript preparation include the following. Conception and design: Hamilton, Gulati. Acquisition of data: Hamilton, Patel, Koltz. Analysis and interpretation of data: all authors. Drafting the article: all authors. Critically revising the article: all authors. Reviewed submitted version of manuscript: all authors. Approved the final version of the manuscript on behalf of all authors: Hamilton. Statistical analysis: Hamilton, Patel, Gulati. Administrative/technical/material support: all authors. Study supervision: Hamilton.

\section{References}

1. Agnelli G, Piovella F, Buoncristiani P, Severi P, Pini M, D'Angelo A, et al: Enoxaparin plus compression stockings compared with compression stockings alone in the prevention of venous thromboembolism after elective neurosurgery. $\mathbf{N}$ Engl J Med 339:80-85, 1998

2. Bernardi E, Camporese G, Büller HR, Siragusa S, Imberti D, Berchio A, et al: Serial 2-point ultrasonography plus D-dimer vs whole-leg color-coded Doppler ultrasonography for diagnosing suspected symptomatic deep vein thrombosis: a randomized controlled trial. JAMA 300:1653-1659, 2008

3. Black PM, Baker MF, Snook CP: Experience with external pneumatic calf compression in neurology and neurosurgery. Neurosurgery 18:440-444, 1986 
4. Brazier J, Roberts J, Deverill M: The estimation of a preference-based measure of health from the SF-36. J Health Econ 21:271-292, 2002

5. Browd SR, Ragel BT, Davis GE, Scott AM, Skalabrin EJ, Couldwell WT: Prophylaxis for deep venous thrombosis in neurosurgery: a review of the literature. Neurosurg Focus 17(4):E1, 2004

6. Chung SB, Lee SH, Kim ES, Eoh W: Incidence of deep vein thrombosis after spinal cord injury: a prospective study in 37 consecutive patients with traumatic or nontraumatic spinal cord injury treated by mechanical prophylaxis. J Trauma 71: 867-871, 2011

7. Danish SF, Burnett MG, Stein SC: Prophylaxis for deep venous thrombosis in patients with craniotomies: a review. Neurosurg Focus 17(4):E2, 2004

8. Dermody M, Alessi-Chinetti J, Iafrati MD, Estes JM: The utility of screening for deep venous thrombosis in asymptomatic, non-ambulatory neurosurgical patients. J Vasc Surg 53: 1309-1315, 2011

9. Ekeh AP, Dominguez KM, Markert RJ, McCarthy MC: Incidence and risk factors for deep venous thrombosis after moderate and severe brain injury. J Trauma 68:912-915, 2010

10. Farray D, Carman TL, Fernandez BB Jr: The treatment and prevention of deep vein thrombosis in the preoperative management of patients who have neurologic diseases. Neurol Clin 22:423-439, 2004

11. Flinn WR, Sandager GP, Cerullo LJ, Havey RJ, Yao JS: Duplex venous scanning for the prospective surveillance of perioperative venous thrombosis. Arch Surg 124:901-905, 1989

12. Flinn WR, Sandager GP, Silva MB Jr, Benjamin ME, Cerullo LJ, Taylor M: Prospective surveillance for perioperative venous thrombosis. Experience in 2643 patients. Arch Surg 131:472-480, 1996

13. Geerts WH, Bergqvist D, Pineo GF, Heit JA, Samama CM, Lassen MR, et al: Prevention of venous thromboembolism: American College of Chest Physicians Evidence-Based Clinical Practice Guidelines. Chest 133 (6 Suppl):381S-453S, 2008

14. Geerts WH, Pineo GF, Heit JA, Bergqvist D, Lassen MR, Colwell CW, et al: Prevention of venous thromboembolism: the seventh ACCP conference on antithrombotic and thrombolytic therapy. Chest 126 (3 Suppl):338S-400S, 2004

15. Gillet JL, Perrin MR, Allaert FA: Short-term and mid-term outcome of isolated symptomatic muscular calf vein thrombosis. J Vasc Surg 46:513-519, 2007

16. Glotzbecker MP, Bono CM, Wood KB, Harris MB: Thromboembolic disease in spinal surgery: a systematic review. Spine (Phila Pa 1976) 34:291-303, 2009

17. Hamilton MG, Hull RD, Pineo GF: Venous thromboembolism in neurosurgery and neurology patients: a review. Neurosurgery 34:280-296, 1994

18. Heit JA, O'Fallon WM, Petterson TM, Lohse CM, Silverstein MD, Mohr DN, et al: Relative impact of risk factors for deep vein thrombosis and pulmonary embolism: a populationbased study. Arch Intern Med 162:1245-1248, 2002

19. Henwood PC, Kennedy TM, Thomson L, Galanis T, Tzanis GL, Merli GJ, et al: The incidence of deep vein thrombosis detected by routine surveillance ultrasound in neurosur- gery patients receiving dual modality prophylaxis. J Thromb Thrombolysis 32:209-214, 2011

20. Iorio A, Agnelli G: Low-molecular-weight and unfractionated heparin for prevention of venous thromboembolism in neurosurgery: a meta-analysis. Arch Intern Med 160:2327-2332, 2000

21. Khaldi A, Helo N, Schneck MJ, Origitano TC: Venous thromboembolism: deep venous thrombosis and pulmonary embolism in a neurosurgical population. Clinical article. J Neurosurg 114:40-46, 2011

22. Kim KS, Brophy GM: Symptomatic venous thromboembolism: incidence and risk factors in patients with spontaneous or traumatic intracranial hemorrhage. Neurocrit Care 11: 28-33, 2009

23. Macdonald PS, Kahn SR, Miller N, Obrand D: Short-term natural history of isolated gastrocnemius and soleal vein thrombosis. J Vasc Surg 37:523-527, 2003

24. Mack WJ, Ducruet AF, Hickman ZL, Kalyvas JT, Cleveland JR, Mocco J, et al: Doppler ultrasonography screening of poor-grade subarachnoid hemorrhage patients increases the diagnosis of deep venous thrombosis. Neurol Res 30:889892, 2008

25. Misra M, Roitberg B, Ebersole K, Charbel FT: Prevention of pulmonary embolism by combined modalities of thromboprophylaxis and intensive surveillance protocol. Neurosurgery 54:1099-1103, 2004

26. Nurmohamed MT, van Riel AM, Henkens CM, Koopman MM, Que GT, d'Azemar P, et al: Low molecular weight heparin and compression stockings in the prevention of venous thromboembolism in neurosurgery. Thromb Haemost 75: 233-238, 1996

27. Ray WZ, Strom RG, Blackburn SL, Ashley WW, Sicard GA, Rich KM: Incidence of deep venous thrombosis after subarachnoid hemorrhage. Clinical article. J Neurosurg 110: 1010-1014, 2009

28. Smith JS, Saulle D, Chen CJ, Lenke LG, Polly DW Jr, Kasliwal MK, et al: Rates and causes of mortality associated with spine surgery based on 108,419 procedures: a review of the Scoliosis Research Society Morbidity and Mortality Database. Spine (Phila Pa 1976) 37:1975-1982, 2012

29. Smith SF, Biggs MT, Sekhon LH: Risk factors and prophylaxis for deep venous thrombosis in neurosurgery. Surg Technol Int 14:69-76, 2005

30. Swann KW, Black PM: Deep vein thrombosis and pulmonary emboli in neurosurgical patients: a review. J Neurosurg 61:1055-1062, 1984

31. Vespa P: Deep venous thrombosis prophylaxis. Neurocrit Care 15:295-297, 2011

Manuscript submitted June 25, 2012.

Accepted November 8, 2012.

Please include this information when citing this paper: published online December 14, 2012; DOI: 10.3171/2012.11.JNS121243.

Address correspondence to: D. Kojo Hamilton, M.D., Department of Neurological Surgery, Oregon Health \& Science University, 3303 SW Bond Avenue, CH8N, Portland, Oregon 97239. email: Kojohamilton1@gmail.com. 